\title{
The AGN nature of three INTEGRAL sources: IGR J18249-3243, IGR J19443+2117, and IGR J22292+6647`
}

\author{
R. Landi ${ }^{1}$, J. B. Stephen ${ }^{1}$, N. Masetti ${ }^{1}$, D. Grupe ${ }^{2}$, F. Capitanio ${ }^{3}$, A. J. Bird ${ }^{4}$, A. J. Dean ${ }^{4}$, M. Fiocchi ${ }^{3}$, and N. Gehrels ${ }^{5}$ \\ 1 INAF/IASF Bologna, via Piero Gobetti 101, 40129 Bologna, Italy \\ e-mail: landi@iasfbo.inaf.it \\ 2 Department of Astronomy and Astrophysics, Pennsylvania State University, University Park, PA 16802, USA \\ 3 INAF/IASF Roma, via Fosso del Cavaliere 100, 00133 Roma, Italy \\ ${ }^{4}$ School of Physics and Astronomy, University of Southampton, Highfield, SO17 1BJ, UK \\ 5 NASA Goddard Space Flight Center, Greenbelt, MD 20771, USA
}

Received 2 July 2008 / Accepted 14 November 2008

\section{ABSTRACT}

\begin{abstract}
Context. The third INTEGRAL/IBIS survey has revealed several new hard X-ray sources, which are still unidentified or unclassified. To identify these sources, we need to find their counterparts at other wavelengths and then study their nature.

Aims. The aim of this work is to employ the capability of the X-ray telescope (XRT) onboard Swift to localise the sources with a positional accuracy of few arcseconds, thus allowing the search for optical/UV, infrared, and radio counterparts to be more efficient and reliable. A second objective is to provide spectral information over a broad range of frequencies.

Methods. We analysed all XRT observations available for three unidentified INTEGRAL sources, IGR J18249-3243, IGR J19443+2117, and IGR J22292+6647, localised their soft X-ray counterparts, and searched for associations with objects in the radio band. We also combined X-/gamma-ray data, as well as all the available radio, infrared, and optical/UV information, in order to provide a broad-band spectral characterisation of each source and investigate its nature.

Results. In all three cases, XRT observations provided a firm localisation of the X-ray counterpart and information on its optical/UV, infrared, and radio associations. All three sources are found to be bright and repeatedly observed radio objects, although poorly studied. The X-/gamma-ray spectrum of each source is described well by power laws with the photon indices typical of AGN; however, only IGR J19443+2117 may have absorption in excess of the Galactic value, while IGR J22292+6647 is certainly variable at X-ray energies. IGR J18249-3243 has a complex radio morphology and a steep radio spectrum, while the other two sources show flatter radio spectra and a more compact morphology. Overall, their radio, optical/UV, and infrared characteristics, as well as their $\mathrm{X}$-/gamma-ray properties, point to an AGN classification for all three objects.
\end{abstract}

Key words. gamma rays: observations - X-rays: galaxies - galaxies: active - galaxies: individual: IGR J18249-3243 galaxies: individual: IGR J19443+2117 - galaxies: individual: IGR J22292+6647

\section{Introduction}

A key strategic objective of the INTEGRAL mission is to survey the sky at high energies $(>20 \mathrm{keV})$, the domain where fundamental changes from primarily thermal to nonthermal sources/phenomena are expected, where the effects of absorption are drastically reduced and where most of the extreme astrophysical behaviour is taking place. To survey the high-energy sky, INTEGRAL makes use of the unique imaging capability of the IBIS instrument, which allows the detection of sources at the mCrab flux level with an angular resolution of $12^{\prime}$ and a point source location accuracy of typically $1-3^{\prime}$ within a large $(29 \times 29$ degrees $)$ field of view.

So far, several surveys produced from data collected by IBIS have been reported in the literature, the most complete being that of Bird et al. (2007), which lists more than 400 sources of various nature (Galactic and extragalactic) and class. However, many $(\sim 25 \%)$ of these new INTEGRAL sources have no obvious counterpart in other wavebands and cannot be firmly classified; their classification is a primary objective of the survey work but it is made difficult by the arcmin size of the IBIS error circles.

* Partly based on observations collected at the Astronomical Observatory of Bologna in Loiano (Italy).
Improved arcsec localization is therefore necessary to pinpoint the X-ray/optical counterpart and through spectroscopic observations assess its nature/class (e.g. Masetti et al. 2008a, and references therein $)^{1}$. Furthermore, many of these IBIS detections are newly discovered X-/gamma-ray emitters and lack spectral information in the $2-10 \mathrm{keV}$ band. Data in this waveband are fundamental to estimate the intrinsic column density via a measurement of the photoelectric cut-off and to have an indication of the source broad-band spectral shape.

Cross correlations with catalogues in other wavebands, in primis radio, can also be employed as a useful tool with which to facilitate the identification process and to identify peculiar and interesting objects. There are many valid reasons to use radio catalogues for counterpart searches: many high energy emitting objects are also powerful radio sources, for example active galactic nuclei (AGNs), pulsars, microquasars and cataclysmic variables $(\mathrm{CVs})$; radio surveys are very sensitive and positionally accurate; the radio band does not suffer from the absorption which may be a limitation in other wavebands particularly for sources in the Galactic plane and center. This is quite important

\footnotetext{
1 The catalogue of INTEGRAL sources identified through optical and near-infrared spectroscopy is available at http: //www . iasfbo . inaf . it/extras/IGR/main.html
} 
Table 1. Log of Swift XRT observations and source position.

\begin{tabular}{|c|c|c|c|c|c|c|}
\hline \multirow[t]{2}{*}{ Source } & \multirow[t]{2}{*}{ 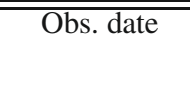 } & \multirow{2}{*}{$\begin{array}{c}\text { Exposure time }^{a} \\
\text { (s) }\end{array}$} & \multirow{2}{*}{$\begin{array}{c}\text { Count rate }(0.3-10 \mathrm{keV}) \\
\left(\text { counts s}{ }^{-1}\right)\end{array}$} & \multicolumn{3}{|c|}{ Position } \\
\hline & & & & $\begin{array}{c}\mathrm{RA} \\
(\mathrm{J} 2000.0)\end{array}$ & $\begin{array}{c}\text { Dec } \\
(\mathrm{J} 2000.0)\end{array}$ & $\begin{array}{c}\text { Error } \\
(\operatorname{arcsec})\end{array}$ \\
\hline IGR J18249-3243 & Apr. 08, 2007 & 4610 & $0.111 \pm 0.005$ & 182456.11 & -324258.9 & 3.7 \\
\hline IGR J19443+2117 & Oct. 10,2006 & 11078 & $0.270 \pm 0.005$ & 194356.20 & +211822.9 & 3.5 \\
\hline IGR J22292+6647 & May 19, 2007 & 9617 & $0.075 \pm 0.003$ & 222913.50 & +664651.8 & 3.6 \\
\hline & May 30, 2007 & 1703 & $0.139 \pm 0.001$ & & & \\
\hline & Jun. 16, 2007 & 1377 & $0.134 \pm 0.001$ & & & \\
\hline & Jul. 10, 2007 & 1656 & $0.147 \pm 0.001$ & & & \\
\hline & Jul. 14, 2007 & $385^{b}$ & $0.092 \pm 0.015$ & & & \\
\hline & Jul. 20, 2007 & 3145 & $0.075 \pm 0.005$ & & & \\
\hline & Aug. 01, 2007 & 2104 & $0.085 \pm 0.006$ & & & \\
\hline & Aug. 02, 2007 & $467^{b}$ & $0.096 \pm 0.015$ & & & \\
\hline
\end{tabular}

${ }^{a}$ Total on-source exposure time;

${ }^{b}$ This observation is not included in the fit procedure because of the too low exposure.

in the case where an optical counterpart is not found, for example due to heavy absorption or when many objects are present within the restricted X-ray error box: radio studies on top of the $\mathrm{X}$-/gamma-ray ones, could help establish the nature of some of these newly discovered INTEGRAL sources.

In this work we provide radio, optical/UV, infrared and $\mathrm{X}$-ray information on three objects extracted from the third IBIS catalogue (IGR J18249-3243, IGR J19443+2117 and IGR J22292+6647) and argue in favour of an AGN nature for all three of them.

\section{X-/gamma-ray data}

All three sources are reported in the third INTEGRAL/IBIS survey (Bird et al. 2007) where position and relative uncertainty, as well as 20-40 and 40-100 keV fluxes can be found. All three are located close to the Galactic plane since IGR J18249-3243, IGR J19443+2117 and IGR J22292+6647 have Galactic latitudes of $-9.2,-1.3$ and +7.7 degrees, respectively. For this work, we use IBIS spectral data obtained following a non standard method, but using the same data set of Bird et al. (2007). The maximum source detection in a single IBIS pointing for all three objects is quite low; consequently, it is not possible to extract a good spectrum from any individual pointing. Instead, for each source all available pointings were processed with the OSA v. 5.1 software to produce images in various energy channels spanning the 20-100 keV range; channels over this band were logarithmically spaced to evenly distribute the counts across the channels. The weighted average flux of the source in each energy channel was then calculated from the individual OSA fluxes and variance images and used to construct a standard spectral pha file. An appropriate rebinned $r m f$ file was produced from the standard IBIS spectral response file to match the chosen energy channels. Here and in the following, spectral analysis was performed with XSPEC v. 11.2.3 (Arnaud 1996) and errors are quoted at $90 \%$ confidence level for one parameter of interest $\left(\Delta \chi^{2}=2.71\right)$.

Due to the low signal to noise ratio of these detections, a simple power law has been employed to fit the IBIS data, providing $\Gamma$ values in the range $\sim 1.5-2.0$.

For each source, we also have X-ray observations acquired with the XRT (X-ray Telescope, 0.2-10 keV, Burrows et al. 2005) onboard the Swift satellite (Gehrels et al. 2004), which allowed us to better determine the position of the IBIS counterpart. XRT data reduction was performed using the XRTDAS standard data pipeline package (XRTPIPELINE v. 0.11.6), in order to produce screened event files. All data were extracted only in the Photon Counting (PC) mode (Hill et al. 2004), adopting the standard grade filtering ( $0-12$ for PC) according to the XRT nomenclature. Images have been extracted in the $0.3-$ $10 \mathrm{keV}$ band and searched for significant excesses falling within or around the INTEGRAL/IBIS $90 \%$ confidence circle as reported in Bird et al. (2007); in all cases a single bright Xray source was detected inside this uncertainty circle or just at its border. The log of all X-ray observations is given in Table 1. The $90 \%$ uncertainty was obtained using the task XRTCENTROID v. 0.2.7. The XRT positions of IGR J182493243 and IGR J19443+2117 are compatible with the location of two ROSAT Bright sources (1RXS J182456.2-324329 and 1RXS J194356.1+211731, respectively). IGR J22292+6647 is instead likely associated with a ROSAT Faint object (1RXS J222915.7+664704) and coincides with a XMMNewton Slew Survey object (XMMSL1 J222914.2+664653); this last measurement also provides a $0.2-12 \mathrm{keV}$ flux of $3 \times$ $10^{-12} \mathrm{erg} \mathrm{cm}^{-2} \mathrm{~s}^{-1}$.

Events for spectral analysis were extracted within a circular region of radius $20^{\prime \prime}$, centered on the source position, which encloses about $90 \%$ of the PSF at $1.5 \mathrm{keV}$ (see Moretti et al. 2004). The background was taken from various source-free regions close to the X-ray source of interest, using circular regions with different radii in order to ensure an evenly sampled background. In all cases, the spectra were extracted from the corresponding event files using the XSELECT v. 2.4 software and binned using GRPPHA, so that the $\chi^{2}$ statistic could be applied. We used version v. 009 of the response matrices and created individual ancillary response files arf using the task XRTMKARF v. 0.5.6.

Only IGR J22292+6647 has more than one pointing; in this case we performed the spectral analysis of those observations having sufficient statistics (6 out of 8 pointings). We found changes in fluxes $(\sim 50 \%)$ but not in shape; we therefore combined measurements with similar count rates to provide a low (LS) and a high (HS) X-ray state for comparison with the average IBIS spectrum.

Next, we analyse the broad-band data of each source over the 0.5-100 keV range. The results of the spectral analysis are listed in Table 2. The best-fits are given by simple power law absorbed by Galactic absorption and, when required, also by adding an intrinsic absorption; we also introduce in the fitting procedure a cross-calibration constant $\left(C_{\text {calib }}\right)$ to account for a possible mismatch between XRT and IBIS data as well as for source flux variations. Only in the case of IGR J19443+2117 do we find evidence for absorption in excess of the Galactic value. The 
Table 2. Best-fit parameters as derived by fitting the XRT and IBIS data.

\begin{tabular}{lcccccccc}
\hline \hline Source & $\begin{array}{c}\text { Energy band } \\
(\mathrm{keV})\end{array}$ & $N_{\mathrm{H}(\mathrm{Gal})}^{a}$ & $N_{\mathrm{H}}^{a}$ & $\Gamma$ & $C_{\text {calib }}^{b}$ & $\chi^{2} / v$ & $F_{(2-10 \mathrm{keV})}^{c}$ & $F_{(20-100 \mathrm{keV})}^{c}$ \\
\hline IGR J18249-3243 & $0.5-100$ & 0.118 & - & $1.54 \pm 0.12$ & $0.52_{-0.21}^{+0.35}$ & $32.7 / 31$ & $0.52 \pm 0.03$ & $0.79 \pm 0.15$ \\
IGR J19443+2117 & $0.9-100$ & 0.836 & $0.54_{-0.13}^{+0.12}$ & $2.04 \pm 0.12$ & $0.60_{-0.28}^{+0.37}$ & $84.3 / 93$ & $1.83 \pm 0.04$ & $1.12 \pm 0.22$ \\
IGR J22292+6647 (low + high) & $1-100$ & 0.481 & - & $1.60 \pm 0.10$ & $0.76_{-0.32}^{+0.45}$ & $120.5 / 105$ & $0.67 \pm 0.05^{e}$ & $1.10 \pm 0.02$ \\
\hline
\end{tabular}

${ }^{a}$ In units of $10^{22} \mathrm{~cm}^{-2}$ (from Kalberla et al. 2005); ${ }^{b}$ IBIS/XRT cross-calibration constant; ${ }^{c}$ in units of $10^{-11} \mathrm{erg} \mathrm{cm}^{-2} \mathrm{~s}^{-1} ;{ }^{d}$ the simultaneous fit of the low and high XRT states, performed by normalising to the low state, provides a cross-calibration constant for the high state of $1.42_{-0.14}^{+0.16}$, thus confirming variability during XRT observations; ${ }^{e}$ in this case we report the average value found for the flux.

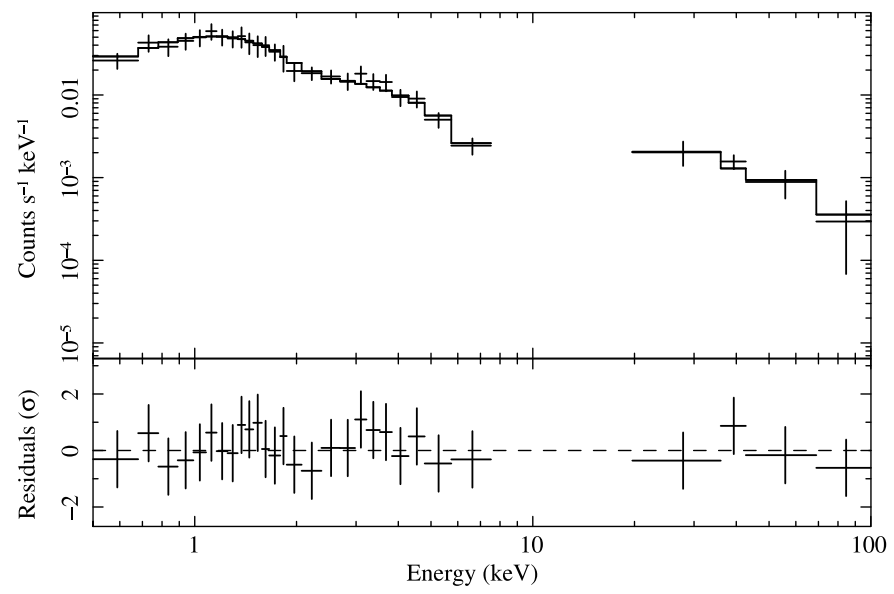

Fig. 1. XRT/IBIS broad-band spectrum of IGR J18249-3243 fitted with a simple power law (upper panel); residuals to this model are in units of $\sigma$ (lower panel).

addition of this component strongly improves the $\chi^{2}$ value, being significant at more than $99.99 \%$ confidence level $\left(\Delta \chi^{2}=73\right.$ for one degree of freedom), according to the $F$-test, and gives a column density of $\sim 8 \times 10^{21} \mathrm{~cm}^{-2}$. Photon indices range again from 1.5 to 1.6, i.e. similar to those typically found in AGNs.

In all sources, the fact that the cross-calibration is different from 1 suggests some flux variability. This is more evident in IGR J22292+6647 due to the larger data set available; indeed in this source XMM-Newton detected a lower X-ray flux (especially in view of the larger band used) than those reported in Table 2. Figures 1-3 show the broad-band X-ray data of each source and, for IGR J22292+6647, also the low and high XRT states (in blue and red in Fig. 3).

\section{Radio data}

Historically AGNs were discovered by radio observations, i.e. the radio selection is often a way to recognize active galaxies, except at lower luminosities where star-formation in galaxies can provide radio emission. Therefore, for bright objects mere detection in radio provides support for the presence of an active galaxy. Contamination from Galactic sources may come from pulsars, microquasars and CVs. In some cases, the radio morphology can help in discriminating between the above possibilities since radio sources showing complex structures (double lobe morphology or hint for jets) are often associated with AGN; furthermore, radio spectra $\left(S \propto v^{\alpha}\right)$ of AGNs are well recognised as being flat $(\alpha=-0.3 /-0.5)$ in compact sources and steep $(\alpha=-0.8 / 1.0)$ in complex objects. Pulsars often have undetected or very dim optical/infrared counterparts, while microquasars are very rare objects. Of the CVs only magnetic systems

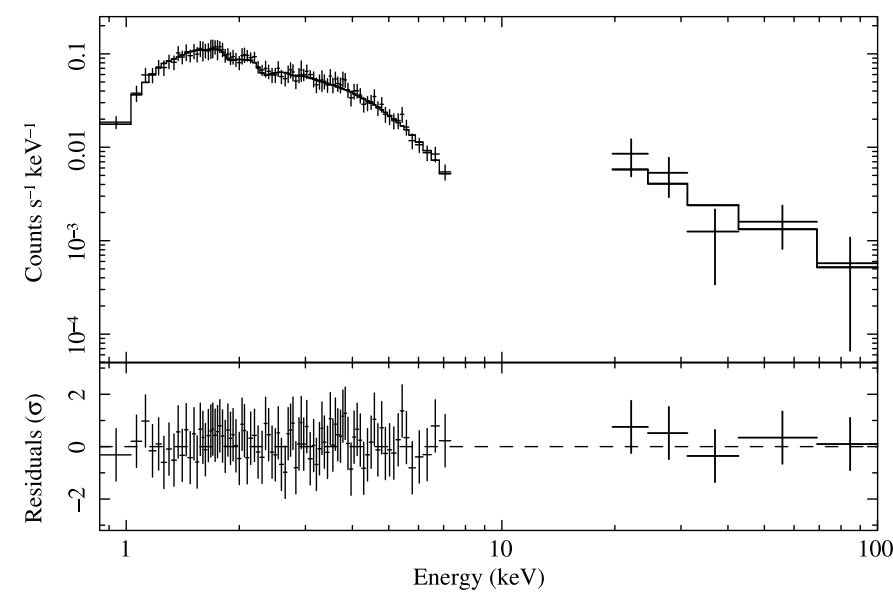

Fig. 2. XRT/IBIS broad-band spectrum of IGR J19443+2117 fitted with an absorbed power law; residuals to this model are in units of $\sigma$ (lower panel).

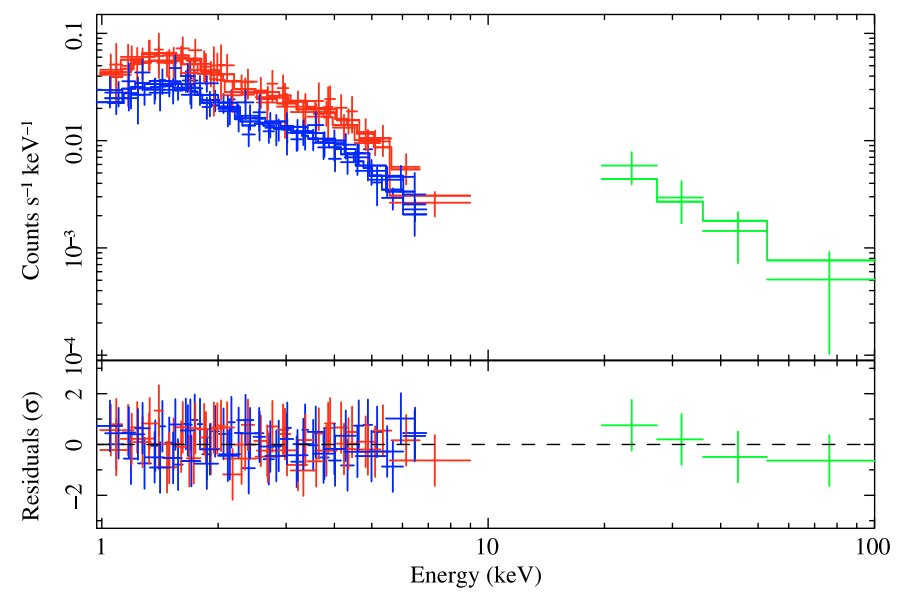

Fig. 3. XRT/IBIS broad-band spectrum of IGR J22292+6647 in low state (blue) and high state (red) fitted with a simple power law; residuals to this model are in units of $\sigma$.

may be associated to radio emission. So, while mere radio detection does not imply identification with an AGN, the combination of high energy X-/gamma-ray emission together with detection in the optical, UV and infrared bands plus complex radio morphology and/or an AGN radio spectrum provide strong support for the AGN nature of an unclassified INTEGRAL source.

For this study, radio images have been taken primarily from the NVSS (NRAO VLA Sky Survey, Condon et al. 1998) at 1.4 GHz. All images have 45 arcsecond FWHM angular resolution and nearly uniform sensitivity. The rms uncertainties in right ascension and declination vary from about 1 arcsecond for the sources stronger than $15 \mathrm{mJy}$ to 7 arcsec at the survey limit 

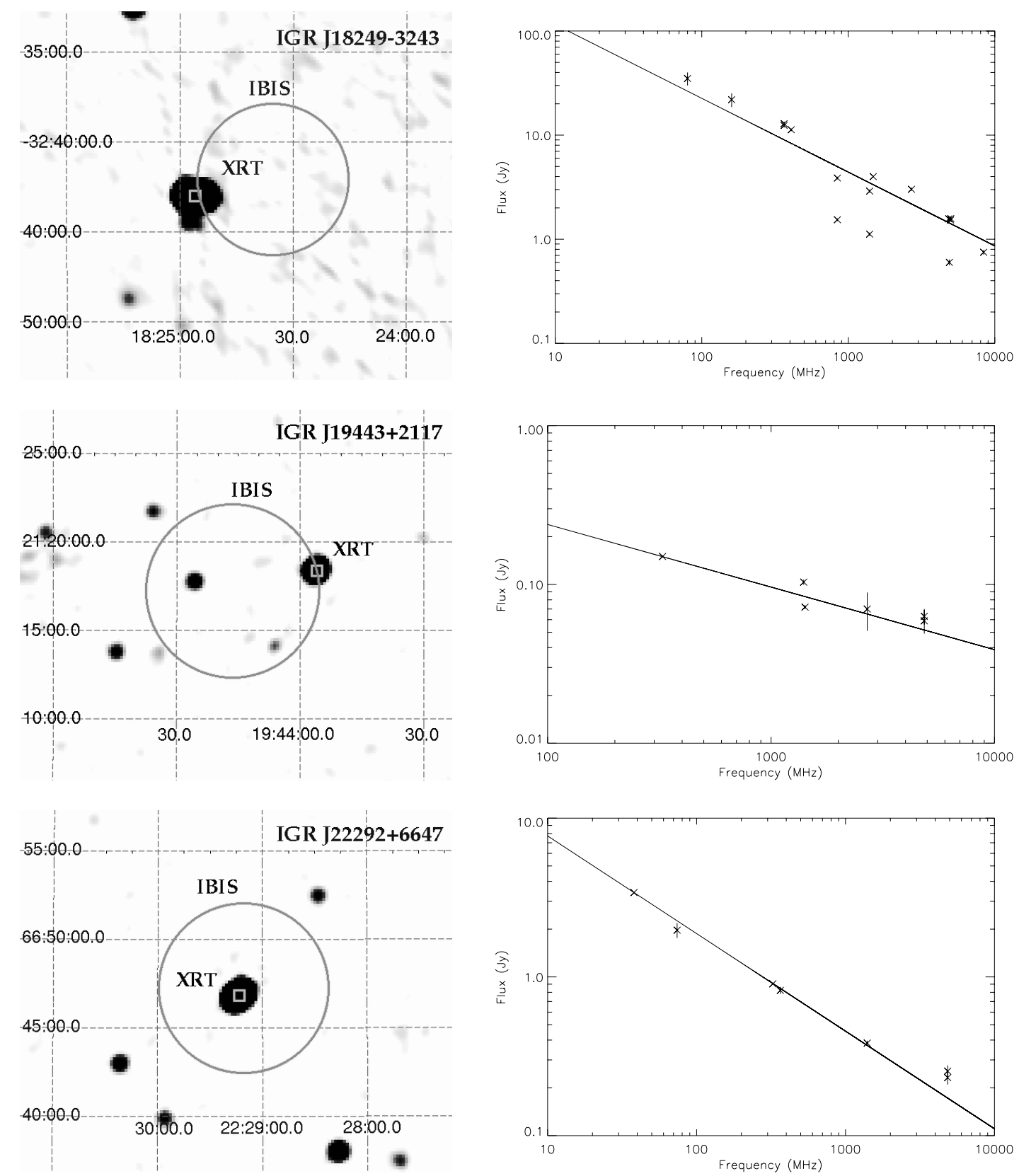

Fig. 4. NVSS images and radio spectra fitted with $\log (S(v))=a \times \log (v)+b$ of IGR J18249-3243 (upper left and right panels), IGR J19443+2117 (central left and right panels) and IGR J22292+6647 (lower left and right panels). The gray circle describes the IBIS uncertainty, while the XRT position is given by the white box.

of $2.5 \mathrm{mJy}$. The left panels of Fig. 4 show the NVSS image cutouts with overimposed IBIS error circle and XRT position. All three objects have, within relative uncertainties, a radio counterpart coincident with the X-ray source. IGR J18249-3243 is a complex source in the NVSS map with at least three components reported (NVSS J182457-324259 with $2.898 \pm 0.101 \mathrm{Jy}$ flux, NVSS J182454-324257 with $1.116 \pm 0.038$ Jy flux, and NVSS J182457-324419 with a $0.0226 \pm 0.0011$ Jy flux); similarly, two very close components are found in the Molonglo Galactic Plane Survey 2nd Epoch (MGPS-2) Compact Source Catalogue (Murphy et al. 2007). The source has been extensively observed at radio frequencies and appears in many radio catalogues, although its nature has been poorly studied so far. It is also reported as an asymmetric double source in NED (see also the Texas Survey of Radio Sources at $365 \mathrm{MHz}$, Douglas et al. 1996). To provide more information on this and the other two sources, we have used SpecFind (Vollmer et al. 2005) and CATS (the on-line Astrophysical CATalogs support System, at http://cats.sao.ru/; see also Verkhodanov et al. 1997); both are tools used to cross-identify radio sources in various catalogues on the basis of self-consistent spectral index as well as position. This allows us to combine data at different frequencies and to estimate the source radio spectrum as $\log (S(v))=$ $a \times \log (v)+b$, where $S$ in expressed in Jy and $v$ in $\mathrm{MHz}$. For IGR J18249-3243 $a$ is $-0.71 \pm 0.01$ and $b$ is $2.77 \pm 0.03$; the corresponding source spectrum is displayed in the upper right panel of Fig. 4.

The structure of IGR J19443+2117 is less complex, with only one component reported as NVSS J194356+211826 with a $1.4 \mathrm{GHz}$ flux of $0.103 \pm 0.004 \mathrm{Jy}$. Also this source appears in various radio catalogues but again its nature and class are not yet defined. Its radio spectrum is plotted in the central right 
Table 3. Summary of the UVOT observations of the three sources. The magnitudes are not corrected for Galactic reddening (Schlegel et al. 1998).

\begin{tabular}{lccc}
\hline \hline Source & Filter & $T_{\exp }$ & Magnitude \\
\hline IGR J18249-3243 & $U V W 2$ & 4727 & $17.53 \pm 0.02$ \\
\hline IGR J19443+2117 & $V$ & 907 & $>20.3(3 \sigma \mathrm{ul})$ \\
& $B$ & 907 & $>21.2(3 \sigma \mathrm{ul})$ \\
& $U$ & 907 & $>20.8(3 \sigma \mathrm{ul})$ \\
& $U V W 1$ & 1826 & $>21.2(3 \sigma \mathrm{ul})$ \\
& $U V M 2$ & 2518 & $>21.5(3 \sigma \mathrm{ul})$ \\
& $U V W 2$ & 1417 & $>21.3(3 \sigma \mathrm{ul})$ \\
\hline IGR J22292+6647 & $U V W 1$ & 11516 & $>21.83(3 \sigma \mathrm{ul})$ \\
& $U V M 2$ & 461 & $>20.41(3 \sigma \mathrm{ul})$ \\
& $U V W 2$ & 5179 & $>21.64(3 \sigma \mathrm{ul})$ \\
\hline
\end{tabular}

Table 4. Summary of the diagnostic parameters.

\begin{tabular}{lcccc}
\hline \hline Source & $\alpha_{\mathrm{ox}}$ & $\alpha_{\mathrm{ro}}$ & $\alpha_{\mathrm{rx}}$ & $\log \left(R_{\mathrm{X}}\right)$ \\
\hline IGR J18249-3243 $^{a}$ & 1.15 & 0.75 & 0.86 & -1.82 \\
IGR J19443+2117 & $>1.38$ & $<0.26$ & 0.63 & -3.74 \\
IGR J22292+6647 & $>1.63$ & $<0.32$ & 0.75 & -2.74 \\
\hline
\end{tabular}

${ }^{a}$ For this source $\alpha_{\text {ox }}$ and $\alpha_{\text {ro }}$ have been estimated by extrapolating the $U V W 2$ flux to $2500 \AA$ and using an average UV slope of $\alpha_{\mathrm{uv}}=0.4$ (Vanden Berk et al. 2001).

panel of Fig. 4 and the spectral parameters in this case are $a=-0.394 \pm 0.016$ and $b=0.165 \pm 0.044$.

IGR J22292+6647 has also one component in the NVSS (NVSS J222913+664654) with a $1.4 \mathrm{GHz}$ flux of $0.381 \pm$ $0.014 \mathrm{Jy}$. Similarly to the other two objects, it is reported in many radio catalogues including the recent VLA Low-Frequency Sky Survey (VLSS, Cohen et al. 2007). The spectral parameters are $a=-0.615 \pm 0.002$ and $b=1.503 \pm 0.003$ and the source radio spectrum is shown in the lower right panel of Fig. 4; also IGR J22292+6647 is reported as an asymmetric double in NED (see also the Texas Survey of Radio Sources at $365 \mathrm{MHz}$ ).

\section{Ultraviolet, optical and infrared data}

All three sources were observed by the Swift UV/Optical telescope (UVOT, Roming et al. 2005). Information regarding UVOT observations are reported in Table 3 . The data were reduced in the standard way by coadding the exposures in each filter by the UVOT task uvotimsum. Source counts were selected with the standard 5 " radius for all UVOT filters according to the most recent UVOT photometry calibration as described by Poole et al. (2008). Background photons were selected in a source-free region close-by with a radius of $20^{\prime \prime}$. Magnitudes were measured with the UVOT tool uvotsource.

Thanks to the spectral information available over a broad range of frequencies, we can determine (see Table 4) the optical-to-X-ray $\left(\alpha_{\text {ox }}=-\log \left(S_{2 \mathrm{keV}} / S_{2500 \AA}\right) / 2.605\right)$, radio-tooptical $\left(\alpha_{\mathrm{ro}}=\log \left(S_{5 \mathrm{GHz}} / S_{2500 \AA}\right) / 5.38\right)$, radio-to-X-ray $\left(\alpha_{\mathrm{rx}}=\right.$ $\left.\log \left(S_{5 \mathrm{GHz}} / S_{2 \mathrm{keV}}\right) / 7.99\right)$ indices and the X-ray radio-loudness parameter $R_{\mathrm{X}}=S_{4.85 \mathrm{GHz}} / S_{2-10 \mathrm{keV}}$ (see Stocke et al. 1991; Terashima \& Wilson 2003). Following Panessa et al. (2007) $\log \left(R_{\mathrm{X}}\right)>-2.755$ can be used as a boundary to determine if an AGN is radio loud.

Within the XRT uncertainty circle of IGR J182493243 , there is a counterpart in the USNO-B1.0 catalogue
(Monet et al. 2003), with $R$ magnitude in the range 14.6-14.8, which is also listed in the 2MASS survey (Skrutskie et al. 2006) with $J=13.456 \pm 0.026, H=13.007 \pm 0.029$, and $K=12.675 \pm$ 0.022 and has a detection in $U V W 2=17.53 \pm 0.02$ (see Table 3 ). The Galactic color excess measured along the line of sight to this object is $E(B-V)=0.23$ (here and afterwards this value is estimated following Schlegel et al. 1998); this value translates to a Galactic column density of $0.13 \times 10^{22} \mathrm{~cm}^{-2}$ (Predehl \& Schmitt 1995), i.e. compatible with the value quoted in Table 2. Recently, optical follow-up observations of IGR J18249-3243 have confirmed the AGN nature of this object despite difficulties in separating the active galaxy at $z=0.355$ from a foreground star (details will be found in Masetti et al. 2008b). It is therefore likely that the USNO-B1.0, 2MASS, and UV magnitudes are contaminated by this star. However, we were able to provide an upper limit to the $R$ flux ${ }^{2}$ of $7.6 \times 10^{-12} \mathrm{erg} \mathrm{cm}^{-2} \mathrm{~s}^{-1}$ (or $6.9 \mathrm{mJy}$ ), adopting the conversion factors of Fukugita et al. (1995). The diagnostic parameters of Table 4 provide additional support for an active galaxy classification for IGR J18249-3243 and further suggest that it might be a radio loud AGN.

Within the XRT error circle of IGR J19443+2117, we find a 2MASS counterpart located at RA $=19^{\mathrm{h}} 43^{\mathrm{m}} 56^{\mathrm{s}} .24$ and Dec $=+21^{\circ} 18^{\prime} 23^{\prime \prime} .4(\mathrm{~J} 2000.0)$, with magnitudes $J>14.53$, $H>13.36$, and $K=13.980 \pm 0.07$. Using the 2MASS conversion factors (Skrutskie et al. 2006), the infrared $K$ flux is $6.5 \times 10^{-13} \mathrm{erg} \mathrm{cm}^{-2} \mathrm{~s}^{-1}$ (or $3.8 \mathrm{mJy}$ ). This infrared source has no counterpart in the USNO-B1.0 catalogue and Swift UVOT images either (see Table 3), implying a dim flux at optical/UV frequencies and difficulties in providing optical spectroscopy in the nearby future. Note also that it is outside the radio error box and may not even be the true counterpart. The Galactic color excess measured in the source direction is $E(B-V)=$ 2.59 , corresponding to a column density of $1.4 \times 10^{22} \mathrm{~cm}^{-2}$ (Predehl \& Schmitt 1995), significantly higher than the Galactic $N_{\mathrm{H}}$ value quoted in Table 2, but compatible with the total absorption measured in this source; this suggests some caution in considering IGR J19443+2117 as an intrinsically absorbed object. The diagnostic parameters listed in Table 4 suggest that IGR J19443+2117 is an AGN more likely of the radio quiet type.

The field of IGR J22292+6647 was observed from Loiano (Italy) with the $1.5 \mathrm{~m}$ "G. D. Cassini" telescope plus BFOSC, equipped with a $1300 \times 1340$ pixels EEV CCD, under nonoptimal weather conditions (seeing: $\left.\sim 3^{\prime \prime}\right)$. The BFOSC images have a plate scale of 0. .58/pix, and a useful field of $13.0 \times 12$. 6 . The imaging observations, performed in the Cousins $R$ band, started at 17:41 UT of 7 November 2007 and were composed of two frames, both with exposure time of $1800 \mathrm{~s}$. Imaging frames were corrected for bias and flat-field in the usual fashion, and were stacked together to improve the signal-to-noise ratio. Visual inspection of the stacked image (see Fig. 5) confirms the presence of an object, also faintly visible on the corresponding DSS-II-Red survey ${ }^{3}$ image, within the XRT error circle. This source appears to be extended, therefore simple aperture photometry, within the MIDAS ${ }^{4}$ package, was used to measure its $R$-band magnitude. This was then calibrated using nearby USNO-A2.0 catalogue ${ }^{5}$ stars. Our final $R$-band photometry of the putative optical counterpart of IGR J22292+6647 yields a

\footnotetext{
${ }^{2}$ Here and in the following the optical and infrared fluxes are corrected for Galactic absorption.

3 Available at http://archive.eso.org/dss/dss

${ }^{4}$ http://www.eso.org/projects/esomidas

5 Available at

http://archive.eso.org/skycat/servers/usnoa/
} 


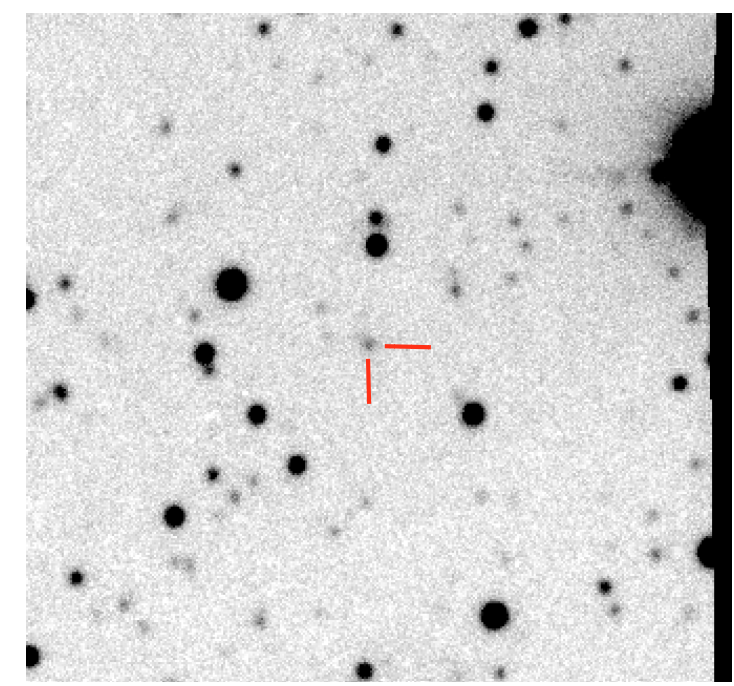

Fig. 5. Section of the "Cassini" plus BFOSC $R$-band stacked image (total exposure time: $3600 \mathrm{~s}$ ) of the field of IGR J22292 +6647 acquired on 7 November 2007. The putative optical counterpart of this high-energy source is indicated by the tick marks. In the image, of size $3^{\prime} \times 3^{\prime}$, North is at top and East is to the left.

magnitude $R=19.2 \pm 0.1$. This object is also listed in the 2MASS survey with magnitudes $J=16.602 \pm 0.143, H=15.707 \pm 0.163$, and $K=14.683 \pm 0.126$. The location of IGR J22292+6647 in the $J-H$ versus $H-K$ color-color diagram is fully compatible with an AGN nature for this source (Spinoglio et al. 1995). The Galactic color excess measured along its line of sight is $E(B-V)=1.089$; this corresponds to a Galactic column density of $0.6 \times 10^{22} \mathrm{~cm}^{-2}$ (Predehl \& Schmitt 1995), i.e. compatible with the value quoted in Table 2. Although the observed $R-K$ value is $\sim 4.5$, i.e. suggestive of a very red object, the corrected value is 2.1 , fully compatible with typical AGN colors. The $R$ magnitude, estimated using the conversion factors of Fukugita et al. (1995), gives a flux, corrected for Galactic absorption, of $8.9 \times 10^{-13} \mathrm{erg} \mathrm{cm}^{-2} \mathrm{~s}^{-1}$ (or $0.8 \mathrm{mJy}$ ). The diagnostic parameters (see Table 4) support an AGN nature for IGR J22292+6647 too and further suggests it might also be a borderline object in between the radio loud and radio quiet regimes.

\section{Conclusions}

We have found the X-ray (and consequently optical and/or $\mathrm{UV} /$ infrared) counterparts of three INTEGRAL unidentified/unclassified sources. The restricted X-ray positions have allowed the association of all three with bright radio emitters often reported in various catalogues but not yet properly studied.

IGR J18249-3243 is the brightest and most complex in radio of the three analysed here and also the farthest away from the Galactic plane; both morphology and spectrum at radio frequencies are compatible with an AGN nature. The X-ray spectrum is flat but still within the range observed in AGN. The diagnostic parameters listed in Table 4 support the AGN classification and also indicate that the source is radio loud. Recent optical spectroscopy confirms our finding locating the source at $z=0.355$ (Masetti et al. 2008b).

The joint XRT and IBIS spectrum of IGR J19443+2117 is also typical of an AGN. The source is moderately bright at radio frequencies and shows a flat spectrum. It is very dim at optical/UV frequencies so that its classification will not be easy; as the source is the closest to the Galactic plane and with the least information available, its classification remains uncertain and therefore we propose it as an AGN candidate, probably radio quiet.

IGR J22292+6647 is the source best studied at high energies: its LS and HS spectra are well fitted with simple power laws having, within the uncertainties, the same photon index but variable fluxes. At radio frequencies the source is quite bright, possibly shows a complex morphology (asymmetric double?) and has a flat spectrum; all these, combined with the infrared photometry and the diagnostic parameters shown in Table 4, are indicative of an AGN nature; it is possible that this source is a borderline object in between the radio loud and radio quiet regimes.

Since optical follow-up spectroscopy of IGR J19443+2117 and IGR J22292+6647 will not be straightforward, we encouraged radio measurements of both objects to confirm our proposed classification.

Acknowledgements. We thank Silvia Galleti and Roberto Gualandi for Service Mode observations at the "Cassini" telescope in Loiano. We also thank Marco Salvati for useful remarks which helped us to improve the paper. This research has made use of data obtained from the High Energy Astrophysics Science Archive Research Center (HEASARC), provided by NASA's Goddard Space Flight Center, the NASA/IPAC Extragalactic Database (NED), the USNO-B1.0 and 2MASS catalogues and the CATS database (Astrophysical CATalogs support System). The authors acknowledge the ASI financial support via ASIINAF grants $\mathrm{I} / 088 / 06 / 0$ and $\mathrm{I} / 008 / 07 / 0$. Swift at PSU is supported by NASA contract NAS5-00136. This research was also supported by NASA contract NNX07AH67G (D.G.). We also acknowledge the use of public data from the Swift data archive.

\section{References}

Arnaud K. A. 1996, XSPEC: The first ten years, in Astronomical Data Analysis Software and Systems V, ed. G. H. Jacoby, \& J. Barnes, ASP Conf Ser., 101, 17

Bird, A. J., Malizia, A., Bazzano, A., et al. 2007, ApJS, 170, 175

Burrows, D. N., Hill, J. E., Nousek, J. A., et al. 2005, Space Sci. Rev., 120, 165 Cohen, A. S., Lane, W. M., Cotton W. D., et al. 2007, AJ, 134, 1245

Condon, J. J., Cotton, W. D., Greisen, E. W., et al. 1998, AJ, 115, 1693

Douglas, J. N., Bash, F. N., Bozyan, F. A., et al. 1996, AJ, 111, 194 Fukugita, M., Shimasaku, K., \& Ichikawa, T. 1995, PASP, 107, 945 Gehrels, N., Chincarini, G., Giommi, P., et al. 2004, ApJ, 611, 1005 Hill, J. E., Burrows, D. N., Nousek, J. A., et al. 2004, Proc. SPIE, 5165, 217 Kalberla, P. M. W., Burton, W. B., Hartmann, D., et al. 2005, A\&A, 440, 775 Masetti, N., Mason, E., Morelli, L., et al. 2008a, A\&A, 482, 113 Masetti, N., Parisi P., Palazzi E., et al. 2008b, A\&A, in press [arXiv: 0811.4085]

Monet, D. G., Levine, S. E., Canzian, B., et al. 2003, AJ, 125, 984

Moretti, A., Campana, S., Tagliaferri, G., et al. 2004, SPIE Proc., 5165, 232

Murphy, T., Mauch, T., Green, A., et al. 2007, MNRAS, 382, 382

Panessa, F., Barcons, X., Bassani, L., et al. 2007, A\&A, 467, 519

Poole, T. S., Breeveld, A. A., Page, M. J., et al. 2008, MNRAS, 383, 627

Predehl, P., \& Schmitt, J. H. M. M. 1995, A\&A, 293, 889

Roming, P. W. A., Kennedy, T. E., Mason, K. O., et al. 2005, Space Sci. Rev., 120,95

Schlegel, D. J., Finkbeiner, D. P., \& Davis, M. 1998, ApJ, 500, 525

Skrutskie, M. F., Cutri, R. M., Stiening, R., et al. 2006, AJ, 131, 1163

Spinoglio, L., Malkan, M. A., Rush, B., Carrasco, L., \& Recillas-Cruz, E. 1995, ApJ, 453, 616

Stocke, J. T., Morris, S. L., Gioia, I. M., et al. 1991, ApJS, 76, 813

Terashima, Y., \& Wilson, A. S. 2002, ApJ, 583, 145

Vanden Berk, D. E., Richards, G. T., Bauer, A., et al. 2001, AJ, 122, 549

Verkhodanov, O. V., Trushkin, S. A., Andernach, H., \& Chernenkov, V. N. 1997,

in Astronomical Data Analysis Software and Systems VI, ed. G. Hunt, \& H.

E. Payne (San Francisco: ASP), ASP Conf. Ser., 125, 322

Vollmer, B., Davoust, E., Dubois, P., et al. 2005, A\&A, 436, 757 